\title{
Neighbourhood walkability and home neighbourhood-based physical activity: an observational study of adults with type 2 diabetes
}

Samantha Hajna ${ }^{1}$, Yan Kestens², Stella S. Daskalopoulou ${ }^{3}$, Lawrence Joseph ${ }^{1,5}$, Benoit Thierry², Mark Sherman ${ }^{6}$, Luc Trudeau ${ }^{4}$, Rémi Rabasa-Lhoret”, Leslie Meissner ${ }^{8}$, Simon L. Bacon ${ }^{9,10}$, Lise Gauvin ${ }^{11}$, Nancy A. Ross ${ }^{1,12}$, Kaberi Dasgupta ${ }^{1,5^{*}}$, Diabetes, GPS, and Walkablilty Study Group

\begin{abstract}
Background: Converging international evidence suggests that diabetes incidence is lower among adults living in more walkable neighbourhoods. The association between walkability and physical activity (PA), the presumed mediator of this relationship, has not been carefully examined in adults with type 2 diabetes. We investigated the associations of walkability with total PA occurring within home neighbourhoods and overall PA, irrespective of location.

Methods: Participants ( $n=97 ; 59.5 \pm 10.5$ years) were recruited through clinics in Montreal (QC, Canada) and wore a GPS-accelerometer device for 7 days. Total PA was expressed as the total Vector of the Dynamic Body Acceleration. PA location was determined using a Global Positioning System (GPS) device (SIRF IV chip). Walkability (street connectivity, land use mix, population density) was assessed using Geographical Information Systems software. The cross-sectional associations between walkability and location-based PA were estimated using robust linear regressions adjusted for age, body mass index, sex, university education, season, car access, residential self-selection, and wear-time.
\end{abstract}

Results: A one standard deviation (SD) increment in walkability was associated with $10.4 \%$ of a SD increment in neighbourhood-based PA (95\% confidence interval (Cl) 1.2, 19.7) - equivalent to 165 more steps/day $(95 \% 19,312)$. Car access emerged as an important predictor of neighbourhood-based PA (Not having car access: $38.6 \%$ of a SD increment in neighbourhood-based PA, $95 \%$ Cl 17.9, 59.3). Neither walkability nor car access were conclusively associated with overall PA.

Conclusions: Higher neighbourhood walkability is associated with higher home neighbourhood-based PA but not with higher overall PA. Other factors will need to be leveraged to facilitate meaningful increases in overall PA among adults with type 2 diabetes.

Keywords: Type 2 diabetes, Physical activity, Accelerometry, Global Positioning Systems, Physical activity locations, Neighbourhood walkability, Environmental epidemiology, Health geography

Abbreviations: BMI, Body mass index; Cl, Confidence interval; GIS, Geographic Information System; GPS, Global Positioning System; PA, Physical activity; SD, Standard deviation; SMARTER, Step Monitoring to Improve ARTERial Health; VeDBA, Vector of the Dynamic Body Acceleration

\footnotetext{
* Correspondence: kaberi.dasgupta@mcgill.ca

'Department of Epidemiology, Biostatistics and Occupational Health, McGill

University, 1020 Pine Avenue West, Montreal, QC, Canada

${ }^{5}$ Department of Medicine, Division of Clinical Epidemiology, McGill University

Health Centre, 687 Pine Avenue West, V1.08, Montréal, QC, Canada

Full list of author information is available at the end of the article
} 


\section{Background}

Adults with type 2 diabetes have low average levels of physical activity $[1,2]$. Even modest increases may lead to important reductions in the risk for diabetes-related complications [3, 4]. It has been suggested that enhancing neighbourhood walkability may help facilitate increases in physical activity, particularly in older adults and/or in those living with chronic conditions [5-7].

Urban planners consider walkable neighbourhoods to be characterized by a variety of services and destinations easily accessed through well-connected street networks $[8,9]$. These emerge when demand for services is high, as in more densely populated areas $[10,11]$. Based on data from general adult populations, residents of such neighbourhoods report higher levels of utilitarian walking (e.g., walking to work) [12, 13]. There is a less consistent relationship between neighbourhood walkability and physical activity assessed objectively (i.e., with biosensor devices such as pedometers and accelerometers). While positive relationships have been delineated in Japan and in some European countries [14], the findings from North American studies are less clear [15, 16]. The relationship between neighbourhood walkability and physical activity has not been well-studied in type 2 diabetes, despite evidence of lower diabetes incidence in more walkable neighbourhoods $[17,18]$.

In the present study, we isolated the subset of total physical activity that occurs within home neighbourhoods and linked this to neighbourhood walkability in a cohort of adults with type 2 diabetes. We hypothesized that a relationship between neighbourhood walkability and physical activity would be more apparent if physical activity occurring specifically within neighbourhoods was considered. Two previous studies have investigated the relationship between neighbourhood walkability and home neighbourhood-based physical activity intensity in adults and demonstrated a positive relationship [19, 20]. We build on this work by examining total levels of physical activity occurring both within home neighbourhoods (excluding inside homes) and overall physical activity irrespective of location in adults with type 2 diabetes.

\section{Methods}

\section{Participants and recruitment procedures}

The study cohort was recruited between November 2012 and February 2015 during the baseline evaluations of an ongoing randomized controlled trial (Step Monitoring to Improve ARTERial Health, SMARTER; NCT0147520) [21]. The objective of SMARTER is to determine if physician-delivered step prescriptions lead to improvements in vascular disease risk among adults with type 2 diabetes or hypertension. Participants were $\geq 18$ years of age at recruitment, under the care of a collaborating physician, and had a body mass index (BMI) between
25 and $40 \mathrm{~kg} / \mathrm{m}^{2}$. Participants with co-morbid conditions that would impede accurate measurement of physical activity (e.g., visual impairments) or adherence to study procedures were excluded from the study. We enrolled SMARTER participants with a physiciandiagnosis of type 2 diabetes and willing to wear an additional unit that combined an accelerometer with a GPS sensor for seven consecutive days as part of their baseline assessment. The SMARTER baseline assessment also included wearing a Yamax SW-701 pedometer with concealed viewing window for seven days. All participants provided written informed consent. Procedures were approved by McGill University's Institutional Review Board (A08-M70-12B) and all participating institutions.

\section{Geographic Information System-derived neighbourhood walkability}

Home neighbourhoods were approximated using 500-m polygonal street network buffers around home addresses using Geographic Information System (GIS) software (ArcMap 10.1; ESRI, Redlands, CA) and digital maps. Street connectivity within each buffer was computed as the number of $\geq 3$-way intersections $/ \mathrm{km}^{2}$. Land use mix was calculated using the entropy formula $(-1) \Sigma_{\mathrm{k}}\left(\mathrm{p}_{\mathrm{k}} \ln \mathrm{n}_{\mathrm{k}}\right) / \ln \mathrm{N}$, where $\mathrm{p}$ represented the proportion of land area devoted to a specific land use $(\mathrm{k})$ in each buffer and $\mathrm{N}$ represented the number of land uses that were being assessed (i.e., four; residential, commercial, institutional/governmental and recreational land uses). Street and land use files were obtained from DMTI CanMap Streetfiles [22]. Population density equaled the number of people per $\mathrm{km}^{2}$ of the census dissemination block where the home was located (2011 Canada Census Population Counts File). A walkability index was calculated by summing the z-scores of the street connectivity, land use mix, and population density measures. A higher index indicated greater walkability.

\section{Location-based physical activity}

Physical activity and location were assessed with researchgrade devices that integrate a GPS monitor (SIRF IV chip) and a tri-axial accelerometer (ADXL 345, Analog Devices) into one unit $(96 \times 80 \times 31.80 \mathrm{~mm}, 125 \mathrm{~g})$. Participants wore the GPS-accelerometer device on their hip for seven days during waking hours, except when showering, bathing, or swimming. They were instructed to connect their unit to a charger every night before going to bed. After the seven-day monitoring period, the device was mailed back to the research center in a postage-paid envelope. Physical activity was expressed as total Vector of the Dynamic Body Acceleration (VeDBA) accumulated over the total valid wear-period. Dynamic Body Acceleration correlates well with the rate of oxygen consumption $[23,24]$. For the purposes of our study, VeDBA (i.e., the dynamic component of body acceleration $\left(\mathrm{m} / \mathrm{s}^{2}\right)$ 
integrated over a one-minute epoch) was summed over each participant's valid wear time. In line with previously established methods $[25,26]$, we retained only individuals with four or more valid wear days (i.e., at least $10 \mathrm{~h}$ of valid data per day). Periods with one hour or more of consecutive accelerometer counts equal to zero were defined as non-wear time.

The GPS-accelerometer devices collected time-stamped latitudes and longitudes at 5-s intervals and raw accelerometer data at $50 \mathrm{~Hz}$ on three axes. The location and accelerometer data were time-matched at the minute level. Participants' homes were identified based on the density and distribution of GPS fixes using a 'hot spot' kernelbased detection algorithm [27]. Each hot-spot was verified to ensure that it matched the residential address that was provided by the participants. Participants with a mismatched home addresses were removed from the analyses. A spatial join was performed between the neighbourhood buffers and the GPS tracks of each participant to identify all GPS coordinates falling within the neighbourhood buffer but outside of the homes. Total VeDBA associated with these "inside neighbourhood" coordinates was computed.

\section{Pedometer-assessed daily steps}

Daily steps were assessed for seven consecutive days at the baseline SMARTER evaluation (Yamax SW-701; viewing windows concealed). Participants were provided with two pedometers. Pedometer A was worn for seven consecutive days. Pedometer B remained in the postage-paid envelope and accounted for extra steps accumulated during the mailing process. Average daily steps were calculated as the number of steps accumulated on Pedometer A minus the number of steps accumulated on Pedometer B divided by the number of days the pedometer was worn. We created a robust linear regression model with which we established the relationship between the number of daily steps and the observed increments in VeDBA.

\section{Covariates}

Season (spring/summer versus fall/winter) was defined based on the evaluation start date. Body mass index (BMI, $\mathrm{kg} / \mathrm{m}^{2}$ ) was computed from weight and height measurements taken by a trained research assistant. The following were queried by questionnaire: age, sex, time since diabetes diagnosis, home address, married/ common-law status, university education, employment, ethnicity, immigrant status, dog ownership, smoking status, insulin use, ownership and/or regular access to a motorized vehicle, depressed mood (Center for Epidemiologic Studies-Depression Scale score $\geq 16$ ) [28], perceived neighbourhood walkability, and the importance of a neighbourhood's walkability when choosing to move there.

\section{Statistical analyses}

Descriptive statistics were produced, overall and by quartile of walkability. Associations between GIS-derived walkability and physical activity were assessed using robust linear regressions ( $\mathrm{m}$ estimation with bisquare weighting) before and after adjustment for the following variables: age, BMI, sex, education, season, car access, residential self-selection and valid wear-time accumulated within neighbourhoods. Higher overall wear-time may allow an individual a greater opportunity to accumulate physical activity. Variables were retained based on theoretical importance and/or if they were identified based on correlation analyses (i.e., $R \geq 0.2$ ) as potential confounders or predictors of neighbourhood-based physical activity. All variables were standardized so that the effect estimates of the linear regression models represented the percent change in 1-standard deviation (SD) of physical activity for a 1-SD increment in the GIS-derived walkability index. We approximated the number of pedometer-assessed daily steps associated with the observed increment in accelerometer-assessed VeDBA using robust linear regression models. To aid in the interpretation of our results, the relationship between BMI across quartiles of neighbourhood walkability was assessed using robust linear regressions. All statistical analyses were conducted using SAS 9.3 (SAS Institute Inc., Cary, NC, USA).

\section{Results}

\section{Characteristics of study population}

Over $70 \%$ of SMARTER participants eligible at the time of recruitment for this study agreed to participate $(156 / 220)$ of whom $71.2 \%$ had $\geq 4$ valid wear days and $62.2 \%$ had complete data on all variables of interest (Fig. 1). Most were married/common-law (69.1\%), university-educated

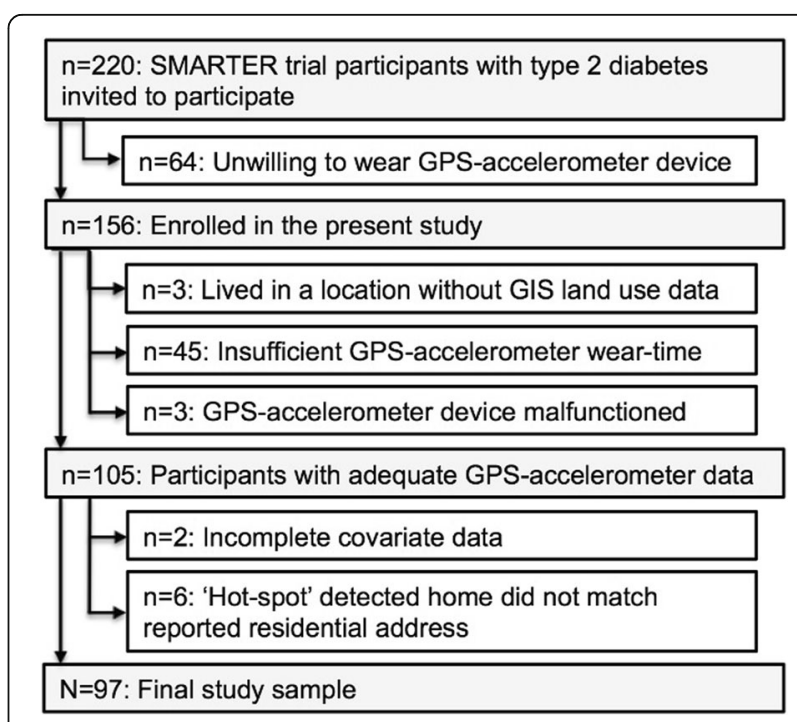

Fig. 1 Selection of study cohort 
(53.6 \%), employed (61.9\%), and lived in the greater Montreal area $(68.0 \%)$. Just over half were men $(56.7 \%)$. The average age was 59.5 years (SD 10.5) and mean BMI was $31.5 \mathrm{~kg} / \mathrm{m}^{2}$ (SD 4.5). On average, participants had diabetes diagnosis for 10.3 years (SD 7.6) and accumulated 4980 steps/day (SD 2798 steps/day). The rates of employment were similar among men (63.6\%) and women (59.5\%). VeDBA occurring anywhere was 615,687 (SD 240,065) and VeDBA occurring specifically within the residential neighbourhoods (excluding at home) was 26,113 (SD 39149).

Neighbourhoods had an average land use mix of 0.3 (SD 0.2), 27 three or more-way intersections $/ \mathrm{km}^{2}$ (SD 14), and 8915 residents $/ \mathrm{km}^{2}$ (SD 8351). Street connectivity, land use mix and population density increased across quartiles of neighbourhood walkability. The least walkable neighbourhoods had an average of 14 intersections per $\mathrm{km}^{2}(\mathrm{SD}=6)$, a land use mix of $0.04(\mathrm{SD}=0.07)$, and a population density of 3920 people per $\mathrm{km}^{2}(\mathrm{SD}=2480)$. In Quartile 2 neighbourhoods there were 26 intersections $/ \mathrm{km}^{2}$ $(\mathrm{SD}=9)$, a land use mix of $0.21(\mathrm{SD}=0.16)$, and population density of 4422 people $/ \mathrm{km}^{2}$ (2421). In Quartile 3 neighbourhoods there were 30 intersections $/ \mathrm{km}^{2}(\mathrm{SD}=8)$, a land use mix of $0.44(\mathrm{SD}=0.15)$, and population density of 8462 people $/ \mathrm{km}^{2}(\mathrm{SD}=5090)$. The most walkable neighbourhoods had an average of 37 intersections per $\mathrm{km}^{2}(\mathrm{SD}=18)$, a land use mix of $0.50(\mathrm{SD}=0.17)$, and a population density of 18,621 people per $\mathrm{km}^{2}$ (9958). Neighbourhood walkability was moderate overall (average GISderived walkability score $=0$, SD 2.15, Range: $-3.5,5.3$ ). The least walkable neighbourhoods (Quartile 1 versus 4) had the highest proportions university education (70.8\% versus $52.0 \%$ ), dog owners (29.2\% versus $12.0 \%)$, and participants with regular car access (91.7 \% versus $64.0 \%)$ (Table 1). No graded patterns were observed for VeDBA across quartiles of neighbourhood walkability. People living in the higher quartiles of neighbourhood walkability placed more importance on the walkability of a neighbourhood in selecting to move to a neighbourhood compared to people living in lower quartiles of neighbourhood walkability. Participants who were excluded from the final analyses (59/156) lived in less walkable neighbourhoods and included a larger proportion of women and a lower proportion of participants who were university educated, employed, immigrants, and/or had depressed mood (see Additional file 1). Those participants who were also excluded due to insufficient valid wear-time (i.e., 45 of these 59) included a larger proportion of individuals who had regular access to a car compared to those who were not excluded $(n=97)$ (i.e., $81.8 \%$ versus $74.2 \%)$ (See Additional file 1).

\section{Multivariate analyses}

Before and after adjustment for age, BMI and sex (Models 1 and 2), small but clinically important associations were observed between neighbourhood walkability and daily steps taken in home neighbourhoods. After further adjustment, these associations remained positive but included possibly clinically unimportant effects. In the fully adjusted model (Model 5) a 1-SD increment in walkability was associated with $10.4 \%$ of a SD increment in neighbourhood-based physical activity (95\% confidence interval (CI) 1.2 to $19.7 \%$; Table 2). This would be similar to taking 165 more steps per day (95\% CI 19 to 312) within home neighbourhoods. No conclusive associations were observed between neighbourhood walkability and overall physical activity (i.e., that occurred anywhere; $0.7 \%$, $95 \%$ CI -13.7 to $15.2 \%$; see Additional file 2).

Not having access to a car emerged as the strongest predictor of higher home neighbourhood-based physical activity after adjustment for factors identified a priori as potential confounders and covariates (Table 3). Those participants who did not have regular car access accumulated $38.5 \%$ of a SD more in home neighbourhoodbased physical activity $(95 \%$ CI $17.9,59.3)$ compared to people who did have regular car access. This is equivalent to an increment of approximately 613 steps per day (95\% CI 284 to 942). No conclusive association was observed between car access and overall levels of physical activity (11.1 \% of a SD increment in neighbourhoodbased physical activity for participants with regular car access compared to participants without regular car access, $95 \%$ CI -21.3 to 43.5 ).

After adjustment for age, sex and education, there was a signaled but inconclusive association between neighbourhood walkability and BMI: Participants who lived in the most compared to the least walkable neighbourhoods (Quartile 4 versus Quartile 1) had a $1.6 \mathrm{~kg} / \mathrm{m}^{2}$ decrement in BMI (95\% CI -4.1 to 0.9 ). This signaled association remained after further adjustment for total physical activity occurring anywhere (i.e., $-1.5 \mathrm{~kg} / \mathrm{m}^{2}$, $95 \% \mathrm{CI}-3.9$ to 1.0$)$.

\section{Discussion}

Our study population achieved an average of 4980 steps/ day, placing them in the "sedentary" category according to the cut-offs proposed by Tudor-Locke [29] and well below the recommended target of 10,000 steps per day [26]. This step count is consistent with the findings of previous studies of adults with type 2 diabetes [2, 7]. Improving neighbourhood walkability has been suggested as a means of facilitating increases in walking [5-7]. Our analyses demonstrate that higher neighbourhood walkability is associated with somewhat higher levels of neighbourhood-based physical activity in adults with type 2 diabetes after adjustment for age, BMI, sex, education, season, car access, and residential self-selection. There was no conclusive evidence, however, that individuals living in walkable neighbourhoods accumulated 
Table 1 Characteristics of study population $(n=97)$

\begin{tabular}{|c|c|c|c|c|c|}
\hline & \multirow{3}{*}{$\begin{array}{l}\text { Overall } \\
\text { Mean (SD) }\end{array}$} & \multicolumn{4}{|c|}{ Quartile of neighbourhood walkability ${ }^{a}$} \\
\hline & & \multirow{2}{*}{$\begin{array}{l}\text { Quartile } 1 \\
\text { Mean (SD) }\end{array}$} & \multirow{2}{*}{$\begin{array}{l}\text { Quartile } 2 \\
\text { Mean (SD) }\end{array}$} & \multirow{2}{*}{$\begin{array}{l}\text { Quartile } 3 \\
\text { Mean (SD) }\end{array}$} & \multirow{2}{*}{$\begin{array}{l}\text { Quartile } 4 \\
\text { Mean (SD) }\end{array}$} \\
\hline & & & & & \\
\hline Age, years & $59.5(10.5)$ & $60.6(12.5)$ & $58.8(7.9)$ & $57.7(11.0)$ & $60.6(10.5)$ \\
\hline Body mass index, $\mathrm{kg} / \mathrm{m}^{2}$ & $31.5(4.5)$ & $32.2(4.9)$ & $32.6(4.9)$ & $30.9(4.0)$ & $30.5(3.9)$ \\
\hline Time since diabetes diagnosis, years & $10.3(7.6)$ & $9.9(8.3)$ & $9.6(8.3)$ & $10.8(5.7)$ & $11.1(8.0)$ \\
\hline Years at current residential address & $18.9(13.9)$ & $22.9(14.1)$ & $15.9(10.8)$ & $20.1(14.4)$ & $17.0(15.7)$ \\
\hline Daily steps, count & 4980 (2798) & $4261(1970)$ & $5957(3214)$ & $4256(2548)$ & $5359(3026)$ \\
\hline Residential self-selection & $0.001(0.93)$ & $-0.48(0.71)$ & $-0.12(0.90)$ & $0.06(0.85)$ & $0.53(0.96)$ \\
\hline Valid wear time, days & $5.9(1.0)$ & $6.0(1.0)$ & $5.8(1.1)$ & $5.6(0.9)$ & $6.0(1.1)$ \\
\hline Total valid monitoring wear-time overall, hours & $86.1(21.1)$ & $90.6(21.0)$ & $84.3(21.8)$ & $83.7(20.3)$ & $85.9(21.7)$ \\
\hline $\begin{array}{l}\text { Total valid monitoring wear-time in } \\
\text { neighbourhoods, hours }\end{array}$ & $1.7(2.8)$ & $0.8(0.5)$ & $1.5(1.3)$ & $2.6(5.3)$ & $1.9(1.5)$ \\
\hline Time in neighbourhood, $\%$ & $1.9(2.4)$ & $0.8(0.6)$ & $1.9(2.1)$ & $2.6(3.7)$ & $2.3(2.0)$ \\
\hline \multicolumn{6}{|l|}{ Total VeDBA } \\
\hline Overall & $615,687(240,065)$ & $601,822(292,986)$ & $619,913(218,942)$ & $611,492(229,354)$ & $628,632(227,385)$ \\
\hline \multirow[t]{2}{*}{ In residential neighbourhoods (excluding home) } & $26,113(39,149)$ & $12,021(13,781)$ & $23,811(27,747)$ & $36,999(67,888)$ & $31,929(24,518)$ \\
\hline & $\%$ & $\%$ & $\%$ & $\%$ & $\%$ \\
\hline Women & 43.3 & 33.3 & 32.0 & 60.9 & 48.0 \\
\hline Married/common-law & 69.1 & 70.8 & 68.0 & 73.9 & 64.0 \\
\hline University education & 53.6 & 70.8 & 60.0 & 30.4 & 52.0 \\
\hline Employed & 61.9 & 58.3 & 64.0 & 65.2 & 60.0 \\
\hline Immigrant & 51.6 & 45.8 & 44.0 & 56.5 & 60.0 \\
\hline Depressed mood & 30.9 & 29.2 & 16.0 & 43.5 & 36.0 \\
\hline Dog ownership & 16.5 & 29.2 & 12.0 & 13.0 & 12.0 \\
\hline Ever smoker & 44.3 & 54.2 & 48.0 & 26.1 & 48.0 \\
\hline Insulin use & 30.9 & 33.3 & 20.0 & 26.1 & 44.0 \\
\hline Car access & 74.2 & 91.7 & 80.0 & 60.9 & 64.0 \\
\hline Spring/summer assessment (versus fall/winter) & 40.2 & 33.3 & 24.0 & 47.8 & 56.0 \\
\hline
\end{tabular}

${ }^{\mathrm{a}}$ Quartile cut-offs for the GIS-derived walkability index: Quartile 1: $<-1.91(n=24)$; Quartile 2: $\geq-1.91<-0.04(n=25)$; Quartile 3: $\geq-0.04<1.40$ ( $n=23$ ); Quartile 4: $\geq 1.40$ ( $n=25)$; Neighbourhood walkability was based on polygonal-shaped buffers

higher levels of overall physical activity (i.e., activity inside the neighbourhoods and elsewhere). Not having regular access to a car was the most important predictor of home neighbourhood-based physical activity.

These findings are consistent with our previous analysis of 2949 Canadian adults who participated in Cycle 1 of the Canadian Health Measures Survey [16], but in contrast to data from Europe and Asia. Our recent meta-analysis of European and Japanese studies which made use of objective measures of neighbourhood walkability and walking showed that adults who live in high compared to low walkable neighbourhoods accumulate overall 766 more steps per day [14]. Socio-environmental contexts may modify the neighbourhood walkabilitytotal physical activity relationship. The beneficial role of neighbourhood walkability on physical activity may be smaller in North America than in Europe/Asia, due to sociocultural differences in physical activity preferences and greater reliance on cars in North America [30].

While some previous studies have demonstrated that not having a car [31, 32], is associated with higher levels of total physical activity, we are the first to show that this factor is associated with greater levels of physical activity occurring specifically within home neighbourhoods. Participants who had regular access to a car achieved approximately 613 fewer steps/day in their home neighbourhoods (95\% CI 284 to 942) than those who did not have regular access to a car. This effect is on par with seasonal deficits in daily steps counts that we observed in another cohort of adults with type 2 diabetes living in Montreal [2]. There we found a deficit of 758 steps per day in the fall/winter compared to the 
Table 2 Regression estimates for the association between neighbourhood walkability and neighbourhood-based total $\operatorname{VeDBA}(n=97)$

\begin{tabular}{lll}
\hline & $\begin{array}{l}\text { Percent change in } \\
\text { one SD of total VeDBA } \\
(95 \% \text { confidence intervals) }\end{array}$ & $\begin{array}{l}\text { Corresponding } \\
\text { change in daily steps } \\
(95 \% \text { confidence intervals })^{c}\end{array}$ \\
\hline Model 1 & $21.2(12.8$ to 29.6$)$ & $337(203$ to 470$)$ \\
Model 2 & $17.6(9.3$ to 26.0$)$ & $280(148$ to 413$)$ \\
Model 3 & $13.9(5.2$ to 22.6$)$ & $221(83$ to 359$)$ \\
Model 4 & $10.0(0.7$ to 19.3$)$ & $159(11$ to 307$)$ \\
Model 5 & $10.4(1.2$ to 19.7$)$ & $165(19$ to 312$)$
\end{tabular}

${ }^{a}$ Model 1: Unadjusted. Model 2: Adjusted for age, BMI, sex. Model 3: Adjusted for age, BMl, sex, university, and season. Model 4: Adjusted for age, BMI, sex, university, season, car access and residential self-selection. Model 5: Adjusted for age, BMI, sex, university, season, car access, residential self-selection and valid wear-time

${ }^{b}$ Estimates represent the percent change in one standard deviation of total VeDBA (95\% confidence interval) occurring within home neighbourhoods (excluding homes) for every one-standard deviation increase in the GIS-derived neighbourhood walkability index. Calculated by multiplying the original estimate by the standard deviation of the walkability index (i.e., 2.16), dividing the result by the SD of the outcome (i.e., 39,149.22) and multiplying by 100

cCalculated using the following formula: daily steps $=-548+0.0089^{*}$ total VeDBA occurring anywhere $)^{*} \%$ change in one SD of VeDBA occurring in neighbourhood/100) where VeDBA occurring anywhere equals one SD of VeDBA occurring anywhere (i.e., 240,065.36)

spring/summer (95\% CI -1037 to -479$)$. An increase of 613 steps per day represents $12.3 \%$ of this group's total daily steps (95 \% CI 5.7 to 18.9 ). With the vast majority of our cohort having regular access to a car, reducing reliance on cars may be an effective way of facilitating increases in neighbourhood-based physical activity among adults with type 2 diabetes, an increase that if sufficient in magnitude might actually increase total physical activity. It is important to note, however, that car access was not conclusively associated with overall physical activity in this population.
International evidence points to remarkably consistent lower diabetes incidence in more walkable neighbourhoods $[17,18,33]$. In a study of 214,882 recent immigrants and 1,024,380 long-term residents living in Toronto (Canada) living in less walkable neighbourhoods (based on population density, residential density, street connectivity, and the availability of retail stores and services) was associated with a higher incidence of diabetes after adjustment for age and area-level poverty (Lowest versus highest walkability quintile; Immigrant men: relative risk [RR] 1.58, 95 \% CI 1.42 to 1.75 , Immigrant women: RR $1.67,95 \% \mathrm{CI} 1.48$ to 1.88 , Long-term resident men: RR 1.32, $95 \%$ CI 1.26 to 1.38 , Long-term resident women: RR $1.24,95 \%$ CI 1.18 to 1.31 ) [17]. Similarly, in an analysis of 512,061 adults living in Sweden, adults who live in the lowest decile of neighbourhood walkability (based on street connectivity, land use mix, and residential density) were found to have a $33 \%$ higher odds of developing incident type 2 diabetes over four years of follow-up (Odds Ratio (OR): 1.33; 95 \% CI 1.13 to 1.55) after adjustment for neighbourhood deprivation [18]. Total physical activity is the presumed link between neighbourhood walkability and diabetes incidence $[17,18]$. It is surprising then that we did not observe an association between neighbourhood walkability and total physical activity. There are several possible explanations for this. First, the positive association between neighbourhood walkability and diabetes incidence may be due to unmeasured variables such as the food environment. More walkable neighbourhoods may have a greater availability of healthy food outlets that may reduce the risk of cardiometabolic complications. This theory is supported by our signaled albeit inconclusive finding that participants living in a more walkable neighbourhood may have lower BMIs than participants living in less walkable neighbourhoods, even

Table 3 Regression estimates for the full-adjusted neighbourhood-based total VeDBA model with corresponding changes in daily steps $(n=97)^{\mathrm{a}}$

\begin{tabular}{|c|c|c|}
\hline & $\begin{array}{l}\text { Percent change in one SD of total VeDBA } \\
\left(95 \% \text { confidence intervals) }{ }^{b}\right.\end{array}$ & $\begin{array}{l}\text { Corresponding change in daily steps } \\
\text { (95\% confidence intervals) }^{c}\end{array}$ \\
\hline Age, years & $-0.01(-0.9$ to 0.8$)$ & $-0.1(-14$ to 14$)$ \\
\hline Women & $-8.5(-26.5$ to 9.4$)$ & $-135(-421$ to 150$)$ \\
\hline Body mass index, $\mathrm{kg} / \mathrm{m}^{2}$ & $-1.2(-3.2$ to 0.9$)$ & $-19(-51$ to 14$)$ \\
\hline University educated (yes versus no) & $-8.8(-26.8$ to 9.1$)$ & $-140(-425$ to 144$)$ \\
\hline Spring/summer assessment (versus fall/winter) & $16.1(-1.2$ to 33.4$)$ & $256(-19$ to 531$)$ \\
\hline Regular car access (yes versus no) & $-38.6(-59.3$ to -17.9$)$ & $-613(-942$ to -284$)$ \\
\hline Residential self-selection score & $5.3(-4.5$ to 15.1$)$ & $84(-72$ to 240$)$ \\
\hline Valid wear-time, minutes & $0.01(-0.002$ to 0.01$)$ & $0.1(-0.03$ to 0.2$)$ \\
\hline
\end{tabular}

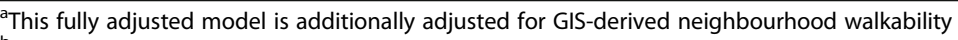

${ }^{b}$ Effect estimates represent the percent change in one standard deviation of total VeDBA occurring in home neighbourhoods (excluding homes) for every one-unit increase in the predictor of interest. Calculated by dividing the original beta estimate by the standard deviation of VeDBA (occurring within home neighbourhoods but excluding homes) (i.e., 39,149.22) and multiplying by 100

cCalculated using the following formula: daily steps $=-548+0.0089^{*}$ total VeDBA occurring anywhere $)^{*}(\%$ change in one SD of VeDBA occurring in neighbourhood/100) where VeDBA occurring anywhere equals one SD of VeDBA occurring anywhere (i.e., 240,065.36) 
after adjustment for total physical activity. Another explanation may be confounding by socioeconomic status. In the Toronto-based study, residual confounding was a possibility since area-level poverty was used as a proxy for individual-level income. This is supported by the fact that the association in the Swedish study was attenuated after additional adjustment for individual-level income as well as age, sex, and education (Adjusted OR: 1.16; 95 \% CI 1.00 to 1.34) [17]. Lastly, it is possible that our exclusion of a large subset of participants due to insufficient wear-time (i.e., $28.8 \%$ ) may have biased our results towards the null. Participants who did not accumulate sufficient wear-time had greater car access (i.e., were likely less active) and lived in less walkable neighbourhoods. Had we been able to include these participants in our analyses a stronger association may have emerged.

There are several strengths to our study. First, we avoided biases arising from participants forgetting to wear one of the devices by using a device that combined an accelerometer and a GPS into one device. This is an improvement over the two previously conducted studies [18], in which participants wore two separate devices. Other strengths include the use of objective measures of walkability and physical activity, and consideration of individual-level covariates and confounders. Some limitations should also be noted. First, our results may not be generalizable to all individuals with type 2 diabetes since only a unique subset of adults with type 2 diabetes may have agreed to participant in this study. Second, $28.8 \%$ of participants did not accumulate enough valid GPS-accelerometer data to be included in the final analyses. Although the mechanism by which these data are missing is unknown, not including these participants in our analyses may have biased our association towards the null as noted above. Third, our sample size limited our ability to assess effect modification. It is possible that factors such as gender or employment status modify the walkability-physical activity relationship. Effect modification by these factors should be assessed in future studies. Lastly, our pedometer-assessed daily steps were not location specific and this means that our daily step estimates represent only approximations of the corresponding changes in VeDBA.

\section{Conclusions}

Our study determined neighbourhood walkability to increase home neighbourhood-based physical activity but no conclusive influence on total activity was discerned in this cohort of adults with type 2 diabetes. Other factors, in combination with walking friendly environments, will need to be leveraged to facilitate meaningful increases in overall PA among adults with diabetes. More research is needed to identify the suite of possible interventions that will facilitate home neighbourhood-based physical activity and ultimately higher levels of overall physical activity.

\section{Additional files}

Additional file 1: Characteristics of the participants that were retained in and excluded from the main analyses. (DOCX $23 \mathrm{~kb}$ )

Additional file 2: Linear regression estimates for the associations between neighbourhood walkability and total VeDBA accumulated anywhere with corresponding changes in daily steps $(n=97)$. (DOCX $17 \mathrm{~kb})$

\section{Declarations}

\section{Acknowledgements}

The authors would like to thank Deborah Chan for her support in data collection, data management, and participant recruitment. The authors would also like to thank Anne-Sophie Brazeau and Corinne Suppère for their help in the recruitment and assessment of participants.

The Diabetes, GPS, and Walkablilty Study Group includes a subgroup of the SMARTER Trial (NCT0147520) collaborators who were invaluable in the recruitment of patients into this study. They include Dr. Sabiha Awan, Dr. Alexis Baass, Dr. Jean-Marie Boutin, Dr. Candace Lee, Dr. Carolina Capelle, Dr. Joanna Caron, Dr. Stavroula Christopolous, Dr. Karen Dahan, Dr. Maxine Dumas-Pilon, Dr. Roy Eappen, Dr. Leah Feldman, Dr. Natasha Garfield, Dr. Dominique Garrell, Dr. Lawrence Green, Dr. Walter Gregory, Dr. Wen Hu, Dr. Sofia Hussaini, Dr. John Hughes, Dr. Catherine Kudo, Dr. April Kinghorn, Dr. Evelyn Kyle, Dr. Leonora Lalla, Dr. Andrea Lalonde, Dr. Pierre Larochelle, Dr. Khue Ly, Dr. Richard Mackler, Dr. Agnieszka Majdan, Dr. Goldie Marmor, Dr. Sara Meltzer, Dr. Hortensia Mircescu, Dr. David Morris, Dr. Kimberly Munro, Dr. Vivian Petropoulos, Dr. Barry Posner, Dr. Brent Richards, Dr. Juan Rivera, Dr. Ghislaine Roederer, Dr. Samantha Sacks, Dr. Alicia Schiffrin, Dr. David Shannon, Dr. Raymond Sorge, Dr. Donald Sproule, Dr. Susan Still, Dr. Robert Wistaff, Dr. Mark Yaffe, Dr. Jean-Francois Yale and Dr. Hans Zingg.

\section{Funding}

Funding for this work was through operating grants from the Heart and Stroke Foundation of Quebec (HSF G-12-000251) and the Canadian Institutes of Health Research (CIHR MOP-79275). SH was supported through a CIHR Doctoral Research Award. KD is supported through a Senior Clinician Scientist Award from the Fonds de Recherché du Québec-Santé. NAR is supported through the Canada Research Chair program (http://www.chairs-chaires.gc.ca).

Availability of data and materials

The Research Ethics (IRB) approvals do not allow for the sharing of these data.

\section{Authors' contributions}

$\mathrm{SH}$ conducted all aspects of the study including participant recruitment, data collection, data management and analyses, the writing of the final manuscript and takes full responsibility for the work as a whole. KD and NAR conceived the study, oversaw all aspects of the study design, provided guidance on the statistical analyses, and reviewed and edited multiple drafts of the manuscript. YK, SSD, SB and LG were involved in the early design phases of this study. $L J$ provided guidance on the statistical analyses. BT converted the raw GPS and accelerometer data unto a usable format. YK and BT and provided guidance in the interpretation of these GPS and accelerometer data. MS, LT and RRL played a key role in the recruitment of participants into this study. All authors reviewed and approved the final manuscript.

\section{Competing interests}

The authors declare that they have no competing interests.

\section{Consent for publication}

Not applicable.

Ethics approval and consent to participate

All participants provided written informed consent. Procedures were approved by McGill University's Institutional Review Board (A08-M70-12B) and all participating institutions.

\section{Author details}

${ }^{1}$ Department of Epidemiology, Biostatistics and Occupational Health, McGill University, 1020 Pine Avenue West, Montreal, QC, Canada. ${ }^{2}$ Centre de 
Recherche du Centre Hospitalier de I'Université de Montréal (CHUM), Tour St-Antoine, S02-340, 850 St-Denis, Montreal, QC, Canada. ${ }^{3}$ Department of Medicine, Division of Internal Medicine, McGill University Health Centre, Montreal General Hospital, 1650 Cedar Avenue, C2.101.4, Montréal, QC, Canada. ${ }^{4}$ Division of Internal Medicine, Jewish General Hospital, 3755 Cote Sainte-Catherine, Montreal, QC, Canada. ${ }^{5}$ Department of Medicine, Division of Clinical Epidemiology, McGill University Health Centre, 687 Pine Avenue West, V1.08, Montréal, QC, Canada. ${ }^{6}$ Department of Medicine, Division of Endocrinology, McGill University Health Centre, 1001 Boulevard Decarie, Montreal, QC, Canada. Institut de Recherches Cliniques de Montréal (IRCM), 110 avenue des Pins, Montréal, QC, Canada. ${ }^{8}$ Department of Medicine, Division of Endocrinology, St. Mary's Hospital, 3830 Lacombe Avenue, Montreal, QC, Canada. ${ }^{9}$ Montreal Behavioural Medicine Centre, Hôpital du Sacré-Cœur de Montréal, 5400 Boul. Gouin Ouest, Montréal, QC, Canada. ${ }^{10}$ Department of Exercise Science, Concordia University, 7141 Sherbrooke Street West, Montreal, QC, Canada. ${ }^{11}$ Département de médecine sociale et préventive, École de Santé Publique, Université de Montréal, 7101 avenue du Parc, Montréal, QC, Canada. ${ }^{12}$ Department of Geography, McGill University, 805 Sherbrooke Street West, Montreal, QC, Canada.

\section{Received: 4 May 2016 Accepted: 27 August 2016}

\section{Published online: 09 September 2016}

\section{References}

1. Tudor-Locke CE, Bell RC, Myers AM, Harris SB, Lauzon N, Rodger NW. Pedometer-determined ambulatory activity in individuals with type 2 diabetes. Diabetes Res Clin Pract. 2002:55:191-9.

2. Dasgupta K, Joseph L, Pilote L, Strachan I, Sigal RJ, Chan C. Daily steps are low year-round and dip lower in fall/winter: findings from a longitudinal diabetes cohort. Cardiovasc Diabetol. 2010;9:81.

3. Gregg EW, Gerzoff RB, Caspersen CJ, Williamson DF, Narayan KM. Relationship of walking to mortality among US adults with diabetes. Arch Intern Med. 2003; 163:1440-7.

4. Yates T, Haffner SM, Schulte PJ, Thomas L, Huffman KM, Bales CW, et al. Association between change in daily ambulatory activity and cardiovascular events in people with impaired glucose tolerance (NAVIGATOR trial): a cohort analysis. Lancet. 2014;383:1059-66.

5. Hirsch JA, Winters M, Ashe MC, Clarke P, McKay H. Destinations that older adults experience within their GPS activity spaces relation to objectively measured physical activity. Environ Behav. 2016;48:55-77.

6. Chaudhury H, Campo M, Michael Y, Mahmood A. Neighbourhood environment and physical activity in older adults. Soc Sci Med. 2016;149:104-13.

7. De Greef K, Van Dyck D, Deforche B, De Bourdeaudhuij I. Physical environmental correlates of self-reported and objectively assessed physical activity in Belgian type 2 diabetes patients. Health Soc Care Community. 2011:19:178-88

8. Saelens BE, Sallis JF, Frank L. Environmental correlates of walking and cycling: Findings from the transportation, urban design, and planning literatures. Ann Behav Med. 2003;25:80-91.

9. Leslie E, Coffee N, Frank L, Owen N, Bauman A, Hugo G. Walkability of local communities: using geographic information systems to objectively assess relevant environmental attributes. Health Place. 2007;13:111-22.

10. Frank LD, Schmid TL, Sallis JF, Chapman J, Saelens BE. Linking objectively measured physical activity with objectively measured urban form: findings from SMARTRAQ. Am J Prev Med. 2005;28:117-25.

11. Frank $L$. Land use and transportation interaction: Implications on public health and quality of life. J Plan Educ Res. 2000;20:6-22.

12. McCormack GR, Shiell A. In search of causality: a systematic review of the relationship between the built environment and physical activity among adults. Int J Behav Nutr Phys Act. 2011;8:125.

13. Van Holle V, Deforche B, Van Cauwenberg J, Goubert L, Maes L, Van de Weghe $N$, et al. Relationship between the physical environment and different domains of physical activity in European adults: a systematic review. BMC Public Health. 2012;12:807.

14. Hajna S, Ross NA, Brazeau A, Bélisle P, Joseph L, Dasgupta K. Associations between neighbourhood walkability and daily steps in adults: A systematic review and meta-analysis. BMC Public Health. 2015;In Press.

15. Oakes JM, Forsyth A, Schmitz KH. The effects of neighborhood density and street connectivity on walking behavior: the Twin Cities walking study. Epidemiol Perspect Innov. 2007;4:16.
16. Hajna S, Ross NA, Joseph L, Harper S, Dasgupta K. Neighbourhood walkability, daily steps and utilitarian walking in Canadian adults. BMJ Open 2015;5, e008964

17. Booth GL, Creatore MI, Moineddin R, Gozdyra P, Weyman JT, Matheson Fl, et al. Unwalkable neighborhoods, poverty, and the risk of diabetes among recent immigrants to Canada compared with long-term residents. Diabetes Care. 2013;36:302-8

18. Sundquist K, Eriksson U, Mezuk B, Ohlsson H. Neighborhood walkability, deprivation and incidence of type 2 diabetes: a population-based study on 512,061 Swedish adults. Health Place. 2015;31:24-30.

19. Rodriguez DA, Brown AL, Troped PJ. Portable global positioning units to complement accelerometry-based physical activity monitors. Med Sci Sports Exerc. 2005;37:S572-81.

20. Troped PJ, Wilson JS, Matthews CE, Cromley EK, Melly SJ. The built environment and location-based physical activity. Am J Prev Med. 2010;38:429-38.

21. Dasgupta K, Rosenberg E, Daskalopoulou SS, Collaborators S. Step Monitoring to improve ARTERial health (SMARTER) through step count prescription in type 2 diabetes and hypertension: trial design and methods. Cardiovasc Diabetol. 2014;13:7.

22. CanMap ${ }^{\oplus}$ Streetfiles. Quebec Land Use Classifications. Markham: DMTI Spatial Inc. 2008. Retrieved from the McGill Univeristy Library.

23. Qasem L, Cardew A, Wilson A, Griffiths I, Halsey LG, Shepard EL, et al. Triaxial dynamic acceleration as a proxy for animal energy expenditure; should we be summing values or calculating the vector? PLoS One. 2012;7, e31187.

24. Halsey LG, Shepard EL, Hulston CJ, Venables MC, White CR, Jeukendrup AE, et al. Acceleration versus heart rate for estimating energy expenditure and speed during locomotion in animals: tests with an easy model species, Homo sapiens. Zoology (Jena). 2008;111:231-41.

25. Hawkins MS, Storti KL, Richardson CR, King WC, Strath SJ, Holleman RG, et al. Objectively measured physical activity of USA adults by sex, age, and racial/ ethnic groups: a cross-sectional study. Int J Behav Nutr Phys Act. 2009;6:31.

26. Colley RC, Garriguet D, Janssen I, Craig CL, Clarke J, Tremblay MS. Physical activity of Canadian adults: accelerometer results from the 2007 to 2009 Canadian Health Measures Survey. Health Rep. 2011;22:7-14.

27. Thierry B, Chaix B, Kestens Y. Detecting activity locations from raw GPS data: a novel kernel-based algorithm. Int J Health Geogr. 2013;12:14.

28. Radloff LS. The CES-D scale: a self-report depression scale for research in the general population. Appl Psychol Meas. 1977;1:385-401.

29. Tudor-Locke C, Hatano Y, Pangrazi RP, Kang M. Revisiting "how many steps are enough?". Med Sci Sports Exerc. 2008;40:S537-43.

30. The World Bank Group. Worldwide Passenger Cars (per 1,000 people). 2007.

31. Lachapelle U, Frank LD. Transit and health: mode of transport, employersponsored public transit pass programs, and physical activity. J Public Health Policy. 2009;30 Suppl 1:S73-94.

32. Shoham DA, Dugas LR, Bovet P, Forrester TE, Lambert EV, Plange-Rhule J, et al. Association of car ownership and physical activity across the spectrum of human development: Modeling the Epidemiologic Transition Study (METS). BMC Public Health. 2015;15:173.

33. Glazier RH, Creatore MI, Weyman JT, Fazli G, Matheson Fl, Gozdyra P, et al. Density, destinations or both? A comparison of measures of walkability in relation to transportation behaviors, obesity and diabetes in Toronto, Canada. PLoS One. 2014;9, e85295.

\section{Submit your next manuscript to BioMed Central and we will help you at every step:}

- We accept pre-submission inquiries

- Our selector tool helps you to find the most relevant journal

- We provide round the clock customer support

- Convenient online submission

- Thorough peer review

- Inclusion in PubMed and all major indexing services

- Maximum visibility for your research

Submit your manuscript at www.biomedcentral.com/submit 